\title{
Occluded Cigarette Smoke Exposure Causing Localized Chloracne-Like Comedones
}

\author{
Andrew T. Patterson ${ }^{a, c}$ Frances T. Tian ${ }^{a}$ Dirk M. Elston ${ }^{d}$ Benjamin H. Kaffenberger ${ }^{b}$ \\ ${ }^{\mathrm{a}}$ Ohio State University College of Medicine, and ${ }^{\mathrm{b}}$ Division of Dermatology, Ohio State University Wexner Medical \\ Center, Columbus, Ohio, 'San Antonio Military Medical Center, San Antonio, Tex., and ${ }^{\mathrm{d}}$ Ackerman Academy of \\ Dermatopathology, New York, N.Y., USA
}

For commentary see p. 294

\section{Key Words}

Acne Environmental hazards .

Occupational skin disease . Smoking

adverse effects - Tobacco smoking .

Occupational exposure $\cdot$ Environmental

acne - Polycyclic aromatic hydrocarbons .

Aryl hydrocarbon receptor - LRIG1 cells

\begin{abstract}
Many environmental acne disorders, including chloracne and oil acne, were previously thought to occur predominantly in occupational settings following polycyclic aromatic hydrocarbon exposure. Cigarette smoke has also been shown to contain a large number of these toxic polycyclic aromatic hydrocarbon components and strictly correlates with noninflammatory acneiform lesion development in postadolescent patients. We report a case of localized open comedones associated with occluded cigarette smoke exposure near the nasal cavity due to infrequently changed gauze following rhinectomy. The dermal uptake of polycyclic aromatic hydrocarbon components in cigarette smoke has the potential to function as a contributing factor in chloracne development. Several of these environmental and noninflammatory acne subtypes may share a common molecular propensity for enhanced comedogenesis originating from aryl hydrocarbon receptor pathway effects in the skin. Additional
\end{abstract}

studies are needed to further elucidate the exact mechanistic pathways through which tobacco smoke impacts the integumentary system.

(c) 2015 S. Karger AG, Basel

\section{Introduction}

Cigarette smoking remains a leading cause of preventable morbidity and mortality in humans and contributes to disease burden in every organ system, including the skin [1]. Commonly described dermatological manifestations associated with chronic smoking may include poor wound healing, melanoma and nonmelanoma skin cancers, oral cancers, psoriasis, eczema, and premature skin aging [2]. Tobacco smoke contains many toxic compounds among its estimated 4,800 constituents, including the class of organic chemicals known as polycyclic aromatic hydrocarbons (PAHs) [3]. Members of this PAH class include various carcinogens and pollutants with pathological effects in humans, including the dioxin and dioxin-like substances implicated in the development of the chloracne subtype of environmental acne $[2,3]$. Chloracne is observed almost exclusively in industrial and occupational settings following exposure to these halogenated aromatic compound 'chloracne- gens' $[4,5]$. Another uncommon form of environmental acne with a similar clinical presentation is pitch or coal tar acne [6]. Several PAH chemicals are present in coal tar-related products, including those utilized in roofing, road paving and other industrial settings $[6,7]$.

Here we present a case of a white male in his 40 s who developed noninflammatory open comedones on the face 1 month after rhinectomy after direct local exposure to cigarette smoke via nasal exhalation and occlusion. This case suggests cigarette smoke exposure as a risk factor for the development of chloracne-like comedones and coal tar acne-like lesions previously thought to occur predominantly in occupational settings.

\section{Case Description}

A 49-year-old Caucasian male with a smoking history of 100 pack-years was initially evaluated for and diagnosed with squamous cell carcinoma of the nasal septum. The patient subsequently underwent extensive surgical intervention, including total rhinectomy, left medial and anterior maxillectomy and radical face and upper lip resections with adjacent tissue rearrangement and lip closure. The patient also completed a course of radiation. Although

\section{KARGER 125}

C 2015 S. Karger AG, Base

$1018-8665 / 15 / 2314-0322 \$ 39.50 / 0$
Benjamin H. Kaffenberger

Ohio State University Wexner Medical Center

915 Olentangy River Road, Suite 4000

Columbus, OH 43212 (USA)

www.karger.com/drm 


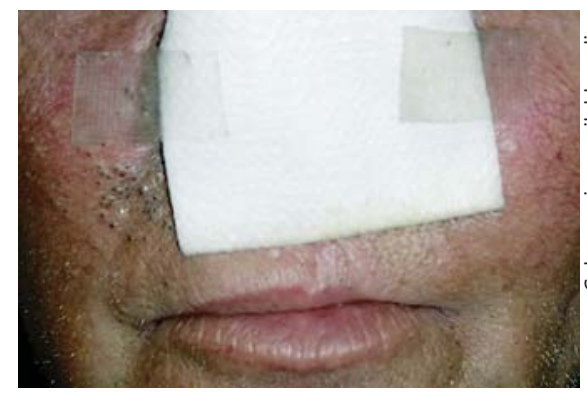

Fig. 1. Localized chloracne. The patient's clinical appearance with evidence of gauze dressing occlusion status after rhinectomy.

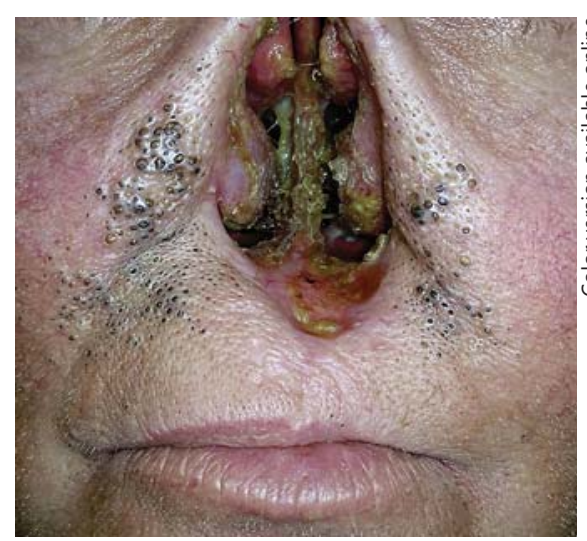

Fig. 2. Localized chloracne. Numerous black noninflammatory open comedones exclusively in the bilateral nasolabial regions occluded by the gauze dressing.

he initially quit, the patient restarted smoking 1.5 packs per day postoperatively while covering the surgical site with gauze (fig. 1).

The following year, the patient was found to have retropharyngeal/parapharyngeal recurrence of the squamous cell carcinoma and underwent further resection and radiative treatments. At this time, the patient was also noted to have numerous black open comedones in the well-demarcated bilateral nasolabial regions that were occluded by the gauze dressing (fig. 2). This prevented reconstruction and he was referred to dermatology. Other than mild pruritus, the lesions were asymptomatic. At the time of examination, the patient reported directly exhaling smoke through his nasal defect while only changing the gauze pad weekly. Based on the lack of occupational or accidental chemical exposures in his history and the distribution of comedones exclusively in areas of direct gauze contact, the etiopathological agent was presumed to be concentrated exposure to halogenated aromatic compounds from the tobacco smoke resulting in the chloracneiform-appearing lesions. The patient was encouraged to quit smoking, change gauze dressings daily, apply benzoyl peroxide topically to the affected areas, and utilize a comedone extractor.

\section{Discussion}

Tobacco smoking causes an overabundance of disease pathology in humans and is implicated in a number of skin diseases [8]. Although a number of studies have examined well-known constituents of cigarette smoke such as nicotine - which has some dermatological anti-inflammatory properties as well as a capacity for stimulating epidermal and mucosal keratinocyte differentiation - the specific effects of the vast majority of the many chemical components of cigarette smoke remain uncharacterized $[9,10]$. While the literature contains mixed data concerning the systemic effect of smoking and its association with acne vulgaris, Capitanio et al. [11] reported a strict correlation with cigarette smoking and the development of comedonal postadolescent acne, a predominantly noninflammatory form of acne characterized by retentional comedones [1, 11-13]. Cigarette smoke has been shown to contain a number of harmful PAHs, including a group of substances further classified as halogenated aromatic compounds [7, 14]. Daily intake of halogenated aromatic compounds in smokers is thought to be consistent with concentrations emitted as flue gas from municipal waste incinerators, with a quantitative estimate of $1-1.6 \mu \mathrm{g}$ of PAHs consumed per cigarette $[7,14]$.

Several halogenated aromatic compound members have been identified as having chloracnegenic potential, including polyhalogenated dibenzofurans, polyhalogenated biphenyls, polyhalogenated naphthalenes, and contaminants of polychlorophenol compounds and related herbicides $[5,8,15]$. Shelley and Kligman [16] were able to experimentally induce localized acne in healthy subjects with daily topical application of penta- and hexachloronaphthalene, further demonstrating the inherently acnegenic potential of these chlorinated organic naphthalene compounds. The dioxin family of halogenated aromatic compounds contains the most potent of the environmental chloracnegens and includes the chemical isomer 2,3,7,8-tetrachlorodibenzo-p-dioxin (TCDD) associated with Agent Orange in the Vietnam War and several industrial disasters $[5,8,17]$. $\mathrm{PAH}$ components have been linked to environmental and occupational acne disorders which can be subclassified into chloracne, pitch acne and oil acne, among others $[4,6]$.

Chloracne is a rare acneiform disorder caused by dioxin absorption via fume inhalation, ingestion or direct skin contact [15]. Chloracne typically presents with numerous noninflammatory open and closed comedones interspersed with straw-colored cysts ranging from 1 to $10 \mathrm{~mm}$ in diameter distributed most frequently in the malar crescent and periauricular regions of the head and neck, with occasional genital and truncal involvement in severe cases [6, 15]. Gray dyschromia, hypertrichosis and follicular hyperkeratosis may accompany the primary lesions [7]. In cases of toxic encounters with dioxins or related halogenated aromatic compounds, skin lesions often function as the sentinel sign of toxicity due to their visibility and preeminent status as a sensitive and specific biomarker for exposure. However, lesional severity is an inconsistent clinical determinant of exposure extent in individual cases $[5,15,18,19]$. Atrisk populations include workers exposed to pesticides, herbicides and other chemical plant manufacturing processes [4]. Though the clinical course is variable, in the majority of cases the initial comedolike lesions appear after a 2- to 4-week latency period following exposure and regress over 6 months to 3 years [8]. As no clinical signs reliably distinguish chloracne from the common exogenous form, the most reliable and specific diagnostic markers are found in the histological disappearance of sebaceous glands and the presence of epidermal cysts [20]. Histology was not performed in our patient due to the cosmetically sensitive nature of the affected facial area. Chloracne is typically refractory to traditional methods of acne treatment, with topical peeling agents and retinoids, oral antibiotics, surgical intervention, and oral retinoids all demonstrating minimal effectiveness [8, 15, 19].

Oil acne is the most common environmental form of acne and is ordinarily observed in machine tool operators, mechanics, refinery workers, and other employees of petroleum-based industries who encounter greases or cutting oils that contain 
a high percentage of mineral oil $[4,6]$. Affected areas commonly include the dorsa of the hands and forearms where direct oil exposure occurs, although truncal areas may be involved with saturation of clothing [4]. Patients frequently develop open comedones initially, with later progression to follicular papules and pustules. Associations with melanosis, photosensitivity and skin carcinomas have also been established [4]. Archetypal oil acne appears less likely in this case due to the absence of inflammatory papules or pustules, lack of typical extremity involvement and low suspicion for source chemical exposure.

Pitch acne is often seen in construction workers, roofers and road pavers and manifests clinically as a purely comedogenic, noninflammatory eruption with a marked predilection for the malar region amongst exposed areas, suggesting an aerosolized mechanism of compound delivery $[4,6]$. Coal tar derivatives are responsible, and these agents include multiple toxic PAHs that possess acnegenic, photosensitizing and carcinogenic properties $[6,7]$.

Another condition with some acneiform components, Favre-Racouchot syndrome (FRS), was also initially considered in the differential diagnosis for this case. FRS is characterized by nodular elastosis with cysts, open comedones and thickened yellowish skin occurring primarily in the temporal and periorbital areas as a result of chronic, extensive actinic damage $[6,21]$. While our patient did not exhibit additional signs of solar damage or possess a typical FRS distribution, it should be noted that a statistically significant dose-related increase in incidence exists for FRS in people with a history of smoking [21]. Smoking has been shown to have a synergistic effect with sun exposure on skin aging, and a similar potentiation effect may contribute to the pathogenesis of this condition associated with extreme solar elastosis $[1,21]$. In light of the proven capacity for airborne $\mathrm{PAH}$ components of coal tar fume elements to cause pitch acne and the demonstrated presence of pathological levels of $\mathrm{PAH}$ chemicals in cigarette smoke, it is not unreasonable to suspect $\mathrm{PAH}$ action on the integumentary systems of these affected patients to partially account for this increased FRS incidence in smokers $[3,6]$.

While the exclusively comedonal nature of pitch acne lesions is used as a point of distinction between the characteristic mixed cystic and comedonal presentation of chloracne, mild cases of chloracne may also manifest as a solely comedogenic condition, which may reflect a shared molecular basis for disease pathophysiology $[4,8$, 15]. Mechanistically, the toxic effects of halogenated aromatic compounds in cigarette smoke are mediated through binding to the aryl hydrocarbon receptor (AhR), which is highly expressed in the human epidermis, hair follicles and sebaceous glands $[3,22,23]$. The AhR ligand complex then migrates to the nucleus where it binds to designated DNA sequences known as xenobiotic-responsive elements in genetic promoter regions and serves to modulate cellular transcription targets [2, 24]. This same AhR receptor also facilitates the dermatological impairments associated with TCDD toxicity in patients with Agent Orange and dioxin exposure and may represent a common mechanistic link among the common forms of occupational and environmental acne [17]. Constitutive activation of these AhRs via chronic PAH exposure is thought to promote immune dysfunction, modifications in vitamin A metabolism and changes in proliferation and differentiation signaling of the epidermis $[3,6]$. While characterizing the precise toxic effects of these AhR agonists can be challenging due to the quantity of $\mathrm{PAH}$ substances present in cigarette smoke, several responsive genomic elements have been identified as being affected, including CYP1A1 and related detoxification enzymes, stress response genes and numerous cell cycle regulators such as the transcriptional repressor B lymphocyte maturation protein 1 (Blimp1) in the sebaceous gland $[3,15,24,25]$. This AhR pathway can be modulated by numerous exogenous and endogenous factors, including food items and the aforementioned many constituents in cigarette smoke, and it is likely that the complex interplay of these influences helps determine the outcome of the comedogenesis in individual patients [26].

With the primary goal of identifying key biological events and potential therapeutic targets at the core of comedogenesis, recent attention has been given to the LRIG1 sebaceous gland stem cells located in the isthmus of the folliculosebaceous unit in the human epidermis [26, 27]. As demonstrated by Saurat [26], these LRIG1 sebaceous gland progenitor cells theoretically regulate differentiation toward an epithelial or sebaceous developmental pathway and therefore are a key target for comedogenic factors such as Propionibacterium acnes bacteria, retinoids, androgens, and local cytokine modulation, among others [26, 27]. These LRIG1 cells have been shown to be highly susceptible to tetrachlorodibenzodioxin/dioxin via the activation of the AhR pathway, ultimately dictating a disproportionate sebogenic differentiation toward an epithelial fate [26]. This response manifests itself clinically by follicular hyperkeratosis, infundibular acanthosis and macrocomedones seen in chloracne and other cases of organochlorine exposures $[18,26]$. In the high-profile case of Victor Yushchenko's massive dioxin poisoning, Saurat and Sorg [20] proposed the labeling of Yushchenko's chloracne-like skin lesions to be called 'metabolizing acquired dioxin-induced skin hamartomas' after finding epidermal cysts with high dioxin concentration, focal expression of the dioxin-metabolizing cytochrome P450 CYP1A1 enzymes, sebaceous gland involution, and reduced lipogenesis $[16,18,20]$.

In summary, this organochlorine-mediated skin toxicity is likely to occur via the LRIG1-regulated alteration of sebaceous gland progenitor cell differentiation into keratinocyte-like developmental pathways through the AhR signaling cascade $[26,28]$. This shared mechanism may account for the overlapping pathogenesis of noninflammatory hyperkeratinization, subsequent obstruction of the pilosebaceous unit and sebaceous gland atrophy that characterizes the comedogenic lesions of chloracne and related disorders $[4,15,20,24]$.

\section{Conclusion}

In the described case, the manifestation of localized facial acneiform lesions was elicited by cigarette smoke occlusion near the patient's nasal cavity due to infrequently changed gauze following rhinectomy. This finding suggests the potential for dermal uptake of these airborne PAHs as a contributing factor in the development of noninflammatory comedonal acne. Furthermore, recent insights into the mechanism of oil acne, pitch acne and chloracne show a common molecular pathway and may represent minor variations linked by the activation of the AhR receptor in the skin.

\section{Disclosure Statement}

The authors have no conflicts of interest or financial disclosures to report. No funding or support was obtained for this study. 


\section{References}

1 Ortiz A, Grando SA: Smoking and the skin. Int J Dermatol 2012;51:250-262.

$>2$ Morita A, Torii K, Maeda A, Yamaguchi Y: Molecular basis of tobacco smoke-induced premature skin aging. J Investig Dermatol Symp Proc 2009; 14:53-55.

3 Kasai A, Hiramatsu N, Hayakawa K, Yao J, Maeda S, Kitamura M: High levels of dioxinlike potential in cigarette smoke evidenced by in vitro and in vivo biosensing. Cancer Res 2006;66:7143-7150.

4 Ancona AA: Occupational acne. Occup Med 1986;1:229-243.

$>5 \mathrm{Ju}$ Q, Zouboulis CC, Xia L: Environmental pollution and acne: chloracne. Dermatoendocrinol 2009; 1:125-128.

6 Adams BB, Chetty VB, Mutasim DF: Periorbital comedones and their relationship to pitch tar: a cross-sectional analysis and a review of the literature. J Am Acad Dermatol 2000;42:624-627.

7 Report on Carcinogens, ed 13. Polycyclic Aromatic Hydrocarbons: 15 Listings. US Department of Health and Human Services, Public Health Service, National Toxicology Program (2014). http://ntp.niehs.nih.gov/go/ roc13.

-8 Tindall JP: Chloracne and chloracnegens. J Am Acad Dermatol 1985;13:539-558.

$>9$ Kwon OS, Chung JH, Cho KH, Suh DH, Park $\mathrm{KC}$, Kim KH, et al: Nicotine-enhanced epithelial differentiation in reconstructed human oral mucosa in vitro. Skin Pharmacol Applied Skin Physiol 1999;12:227-234.

10 Kalayciyan A, Orawa H, Fimmel S, Perschel FH, González JB, Fitzner RG, et al: Nicotine and biochanin $\mathrm{A}$, but not cigarette smoke, induce anti-inflammatory effects on keratino- cytes and endothelial cells in patients with Behçet's disease. J Invest Dermatol 2007;127: 81-89.

11 Capitanio B, Sinagra JL, Bordignon V, Cordiali FP, Picardo M, Zouboulis CC: Underestimated clinical features of postadolescent acne. J Am Acad Dermatol 2010;63:782788 .

12 Schafer T, Nienhaus A, Vieluf D, Berger J, Ring J: Epidemiology of acne in the general population: the risk of smoking. Br J Dermatol 2001;145:100-104.

$>13$ Klaz I, Kochba I, Shohat T, Zarka S, Brenner S: Severe acne vulgaris and tobacco smoking in young men. J Invest Dermatol 2006;126: 1749-1752.

14 Muto H, Takizawa Y: Dioxins in cigarette smoke. Arch Environ Health 1989;44:171174.

15 Panteleyev AA, Bickers DR: Dioxin-induced chloracne - reconstructing the cellular and molecular mechanisms of a classic environmental disease. Exp Dermatol 2006;15:705730 .

16 Shelley WB, Kligman AM: The experimental production of acne by penta- and hexachloronaphthalenes. AMA Arch Derm 1957;75: 689-695.

-17 Patterson AT, Kaffenberger BH, Keller RA, Elston DM: Skin diseases associated with Agent Orange and other organochlorine exposures. J Am Acad Dermatol 2015, Epub ahead of print.

18 Saurat JH, Kaya G, Saxer-Sekulic N, Pardo B, Becker M, Fontao L, et al: The cutaneous lesions of dioxin exposure: lessons from the poisoning of Victor Yushchenko. Toxicol Sci 2012;125:310-317.
19 Ju Q, Zouboulis CC: Effects of 2,3,7,8-tetrachlorodibenzo- $p$-dioxin on the skin. Biosafety $2013 ; 2: 1000112-1000114$

20 Saurat JH, Sorg O: Chloracne, a misnomer and its implications. Dermatology 2010;221: 23-26.

21 Keough GC, Laws RA, Elston DM: Favre-Racouchot syndrome: a case for smokers' comedones. Arch Dermatol 1997;133:796-797.

22 Furue M, Takahara M, Nakahara T, Uchi H: Role of AhR/ARNT system in skin homeostasis. Arch Dermatol Res 2014;306:769-779.

$23 \mathrm{Ju}$ Q, Yu Q, Song N, Tan Y, Xia L, Zouboulis $\mathrm{CC}$ : Expression and significance of aryl hydrocarbon receptor on human epidermis, hair follicle, and sebaceous gland. Chin J Dermatol 2011;44:761-764.

24 Ono Y, Torii K, Fritsche E, Shintani Y, Nishida E, Nakamura M, et al: Role of the aryl hydrocarbon receptor in tobacco smoke extract-induced matrix metalloproteinase-1 expression. Exp Dermatol 2013;22:349-353.

25 Ikuta T, Ohba M, Zouboulis CC, Fujii-Kuriyama Y, Kawajiri K: B lymphocyte-induced maturation protein 1 is a novel target gene of aryl hydrocarbon receptor. J Dermatol Sci 2010;58:211-216.

26 Saurat JH: Strategic targets in acne: the comedone switch in question. Dermatology 2015; 231:105-111.

27 Barnes L, Puenchera J, Saurat JH, Kaya G: LRIG1 and CD44v3 expression in the human folliculosebaceous unit. Dermatology 2015; 231:116-118.

28 Ju Q, Fimmel S, Hinz N, Stahlmann R, Xia L, Zouboulis CC: 2,3,7,8-Tetrachlorodibenzo- $p$ dioxin alters sebaceous gland cell differentiation in vitro. Exp Dermatol 2011;20:320-325. 